\title{
Low-energy electronic states of carbon nanocones in an electric field
}

\author{
Jun-Liang Chen ${ }^{1}$, Ming-Horng Su², Chi-Chuan Hwang, ${ }^{3,}$, Jian-Ming Lu and Chia-Chang Tsai ${ }^{5}$
}

The low-energy electronic states and energy gaps of carbon nanocones in an electric field are studied using a single- $\pi$-band tight-binding model. The analysis considers five perfect carbon nanocones with disclination angles of $60^{\circ}, 120^{\circ}, 180^{\circ}, 240^{\circ}$ and $300^{\circ}$, respectively. The numerical results reveal that the low-energy electronic states and energy gaps of a carbon nanocones are highly sensitive to its geometric shape (i.e. the disclination angle and height), and to the direction and magnitude of an electric field. The electric field causes a strong modulation of the state energies and energy gaps of the nanocones, changes their Fermi levels, and induces zero-gap transitions. The energy-gap modulation effect becomes particularly pronounced at higher strength of the applied electric field, and is strongly related to the geometric structure of the nanocone.

Keywords: Low-energy electronic states; Carbon Nanocones; Electric field

Citation: Jun-Liang Chen, Ming-Horng Su, Chi-Chuan Hwang, Jian-Ming Lu and Chia-Chang Tsai, "Low-energy electronic states of carbon nanocones in an electric field", Nano-Micro Lett. 2, 121-125 (2010). doi:10.5101/nml. v2i2.p121-125

The properties and potential applications of carbon-related nanometer-size materials have attracted intensive interest in recent years. Of these various materials, carbon nanotubes play a particularly crucial role in the field of nano-material science and technology. Since the observation of multiwalled carbon nanotubes by Iijima [1], many carbon nanostructures have identified, such as carbon tori [2], carbon nanohorns [3], and carbon nanocones [4], etc. A graphite sheet can be notionally divided into six equivalent wedges by choosing one of its hexagon centers through each of its corners. A nanocone can then be formed by removing one or more of these wedges from the graphite sheet and rolling up the remainder. Nanocones are identified by their disclination angle $\alpha$ where this angle corresponds to the angle of the part removed from the sheet before the rolling process. The surface of a nanocone can be regarded as a hexagonal network containing pentagonal defects.
The growth process of carbon nanocones compared to those of carbon nanotubes and $\mathrm{C}_{60}$ was studied by Ge and Sattler [4]. The results showed that perfect carbon nanocones have one of five possible open apex angles, i.e. $123.6^{\circ}, 86.6^{\circ}, 60^{\circ}, 38.9^{\circ}$ and $19.2^{\circ}$ corresponding to their disclination angles of $60^{\circ}$, $120^{\circ}, 180^{\circ}, 240^{\circ}$ and $300^{\circ}$, respectively.

Many theoretical [5-10] and experimental studies [11-14] of the geometric and electronic properties of carbon nanocones or nanotube tips have been revealed over the past decade. In general, the results have shown that the electronic structure, e.g. the electronic states, energy gaps and density of states, are highly sensitive to the symmetry of the edge sites at the open apex and to the curvature effect. However, relatively few studies have investigated the effect of an electric field on the electronic structures of carbon nanocones. It is known that an electric field can significantly affect the electronic properties of

${ }^{1}$ Assistant Professor, Department of Mechanical Engineering, WuFeng Institute of Technology

2Professor, Department of Fire Science, WuFeng Institute of Technology

${ }^{3}$ Professor, Department of Engineering Science, National Cheng Kung University

${ }^{4}$ Associate Researcher, Advanced Networking Business Unit, National Center for High-Performance Computing, National Applied Research Laboratories

5 Postdoctoral Researcher, Institute of Atomic and Molecular Sciences, Academia Sinica

*Corresponding author. Email: chchwang@mail.ncku.edu.tw. Tel: (+886)-6-2757575 ext. 63348; Fax: (+886)-6-2766549 
carbon-related systems such as carbon nanotubes [15-21], carbon tori [22], and layered graphite [23]. Hence, it is reasonable to speculate that the energy gap of a carbon nanocone will also be modulated under the effects of a uniform electric field. Accordingly, in this study, a tight-binding model is employed to investigate the changes, induced by a uniform electric field, on the low-energy electronic structures of perfect carbon nanocones.

The schematic diagram of a carbon nanocone is shown in Fig. 1. The current investigations consider carbon nanocone structures with disclination angles $\alpha$ of $60^{\circ}, 120^{\circ}, 180^{\circ}, 240^{\circ}$ and $300^{\circ}$, respectively. Each of these nanocones is comprised a similar number $N$ of carbon atoms. The $\mathrm{C}-\mathrm{C}$ bond length $a_{\mathrm{C}-\mathrm{C}}$ of this conical system is approximately $1.42 \AA$, and the open base of each nanocone is terminated by hydrogen atoms. The single- $\pi$-band tight-binding model is used to calculate the low-energy electronic states. In this model, the $2 p_{z}$ orbitals can describe completely the $\pi$-electronic states so as to dominate exactly the low-energy electronic properties. However, the $\sigma$ bands are omitted because they are far from the Fermi energy $E_{F}$. In present analysis, the interaction between two nearest-neighbor carbon atoms is considered and the magnitude of this interaction, $\gamma_{0}$, is assumed to be $3.033 \mathrm{eV}$ [24]. When an electric field $\mathbf{E}$ is applied, the low-energy electronic state of the nanocones is modulated drastically. However, it is assumed that $\mathbf{E}$ has no effect on the geometric structure of the nanocones and thus on the parameter $\gamma_{0}$. Furthermore, due to the screen effect, $\mathbf{E}$ is regarded as an effective field, that is, it changes only the on-site potential of the carbon atoms.

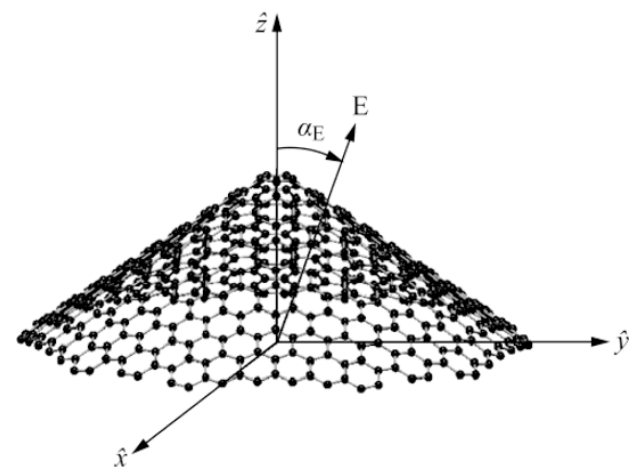

FIG. 1. Schematic diagram of carbon nanocone showing Cartesian coordinate system and a uniform electric field, $\mathbf{E}$, applied at an incidence angle, $\alpha_{E}$, to the cone axis ( $(\hat{z}$-axis)

The nearest-neighbor Hamiltonian is given by

$$
H=\sum_{i=1}^{N} \varepsilon_{i}(\mathbf{E}) c_{i}^{\dagger} c_{i}+\sum_{\substack{i, j=1 \\ i \neq j}}^{N}\left(-\gamma_{0}\right) c_{i}^{\dagger} c_{j}
$$

where $\varepsilon_{i}(\mathbf{E})$ are the unperturbed on-site potentials induced by $\mathbf{E}$, and $c_{i}^{\dagger}\left(c_{i}\right)$ the creation (annihilation) operators of Fermions. The Hermitian matrix representation of this Hamiltonian is built from the subspace spanned by the $N$ basis wavefunctions of $2 p_{z}$ orbitals. For convenience, the Cartesian coordinate is used in current computations and the position of each atom is denoted as $\left(x_{i}, y_{i}, z_{i}\right), i=1, \cdots, N$. The origin of the coordinate is located at the center of the carbon nanocone (see Fig. 1). The symmetry axis of the carbon nanocone is parallel to the $\hat{z}$-axis of the coordinate. The distance $\Delta_{i j}$ between the $i$-th and $j$-th carbon atoms is given by

$\Delta_{i j}=\sqrt{(\Delta x)^{2}+(\Delta y)^{2}+(\Delta z)^{2}}$

Where $\Delta x=\left|x_{i}-x_{j}\right|, \Delta y=\left|y_{i}-y_{j}\right|$ and $\Delta z=\left|z_{i}-z_{j}\right|$. If $\mathbf{E}$ is absent, the elements of the Hamiltonian matrix are given by

$H_{i j}=\left\{\begin{array}{cl}-\gamma_{0} & \Delta_{i j}=a_{\mathrm{C}-\mathrm{C}} \\ 0 & \text { otherwise }\end{array}\right.$

However, when the electric field is applied, the on-site energies of the carbon nanocone will be changed. In present analysis, the electric field is assumed to be

$\mathbf{E}=E \cos \alpha_{E} \mathbf{e}_{\hat{z}}+E \sin \alpha_{E} \mathbf{e}_{\hat{x}}=\mathbf{E}_{\|}+\mathbf{E}_{\perp}$

Where, $E$ is the strength of the electric field and in the unit of $\gamma_{0} / \AA$. Meanwhile, $\alpha_{E}$ is the angle between the electric field direction and the $\hat{z}$-axis, and $\mathbf{e}_{\hat{z}}\left(\mathbf{e}_{\hat{x}}\right)$ is the unit vector in $\hat{z}$-axis ( $\hat{x}$-axis). After applying the electric field, the diagonal elements of the Hamiltonian matrix vary from zero to

$H_{i i}=\varepsilon_{i}(\mathbf{E})=-E z_{i} \cos \alpha_{E}-E x_{i} \sin \alpha_{E}$

The electronic state energies of carbon nanocones are obtained by diagonalizing the $N$ by $N$ Hamiltonian matrix [25-28], and are expressed as $E^{c, v}(M, \mathrm{E})$, where $M$, from 1 to $N$, represents the discrete state, and $c(v)$ corresponds to the unoccupied (occupied) states. Note that an energy gap, $E_{g}$, may exist between the highest occupied molecular orbital (HOMO) state and the lowest unoccupied molecular orbital (LUMO) state.

The present analysis commences considering the effects on the low-energy electronic states of the carbon nanocones due to an electric field whose direction is parallel to the symmetry axis of the cone, i.e. $\mathbf{E}=\mathbf{E}_{\|}=E \mathbf{e}_{\hat{z}}$ and $\alpha_{E}=0^{\circ}$. Figure 2(a) (e) presents the $\pi$-electronic states corresponding to the $2 p_{z}$ orbitals of the five nanocones. Note that the value of Fermi energy is set to be zero $\left(E_{F}=0\right)$ in the computations. At $E=0$, it 

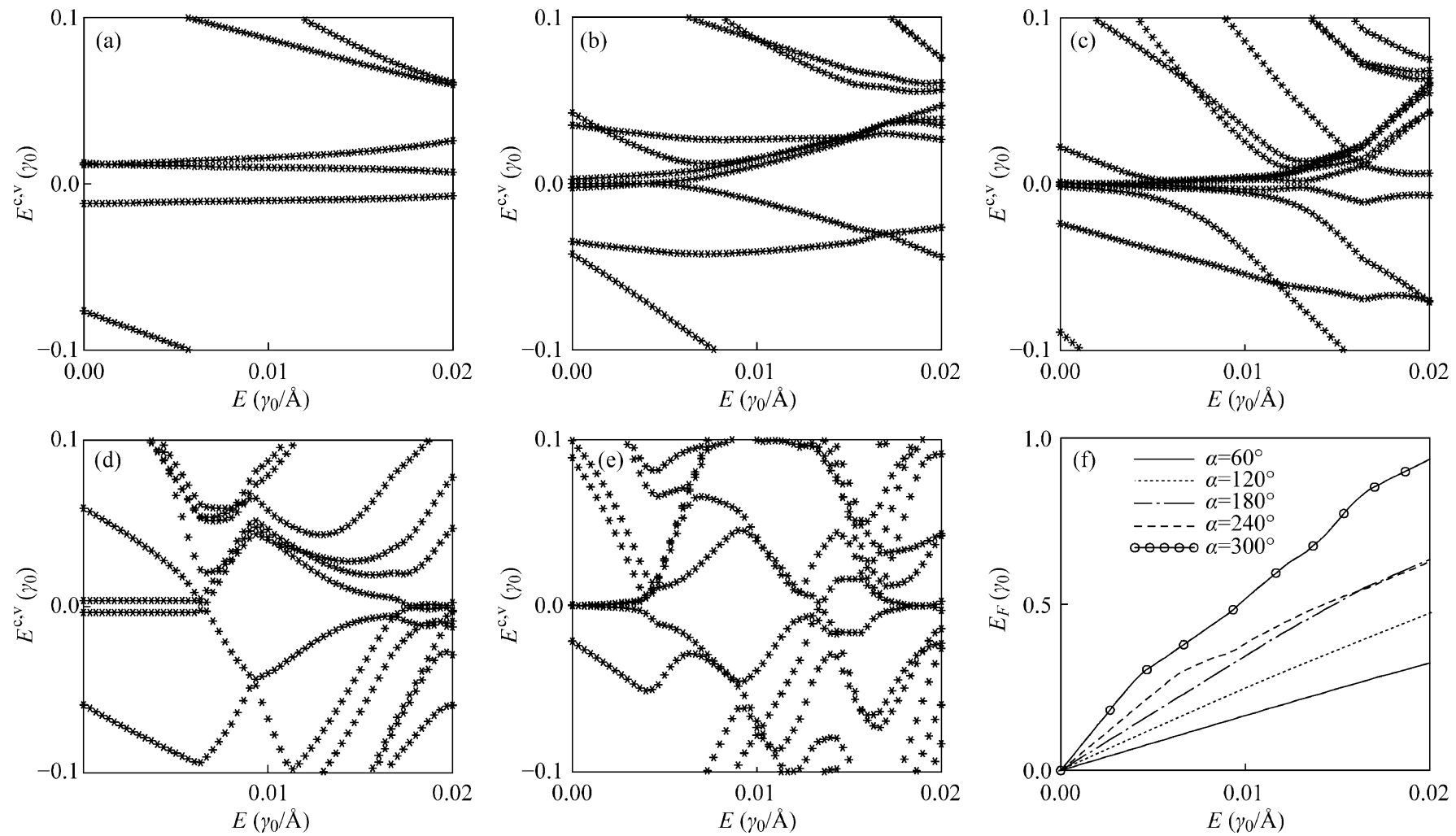

FIG. 2. Electric-field-dependent low-energy states of carbon nanocones for $\alpha_{E}=60^{\circ}$ : (a). $N=1080, \alpha=60^{\circ},(\mathrm{b}) . N=1060, \alpha=120^{\circ}$, (c). $N=1050, \alpha=180^{\circ},(\mathrm{d})$. $N=1056, \alpha=240^{\circ}$, (e). $N=1022, \alpha=300^{\circ}$, where $N$ is the total number of carbon atoms in the nanocone and $\alpha$ is the disclination angle. (f). Electric-field-dependent Fermi energies corresponding to five systems shown in (a) to (e).

is observed that the lowest electronic states, which are close to $E_{F}$, are symmetric, while the other states are non-symmetric. However, as $E$ increases, the electronic states are drastically modulated and state crossings take place at $E_{F}=0$ indicating the occurrence of zero-gap transitions (ZGTs), i.e. a change in the energy gap from a finite value to zero, or vice versa. It is revealed that the ZGTs occur more frequently at larger $E$ values. The nanocone with the largest disclination angle, $\alpha=300^{\circ}$, has the greatest height between the apex and the base, and the magnitude of the on-site potential induced by $\mathbf{E}_{\|}$is also the highest. As a result, a more drastic modulation of the electronic states takes place. In other words, as the strength of the applied electric field or the disclination angle increases, the extent of the modulation effect also increases. Significantly, the HOMOs and LUMOs of the carbon nanocones are symmetric to one another about $E_{F}$ at larger $E$ values. Finally, Fig. 2(f) shows that the Fermi energies of these five systems increase almost linearly with increasing $E$ (larger $E$ indicates the increment of the on-site potential $\varepsilon_{i}(\mathbf{E})$ ). Overall, the results presented in Fig. 2 demonstrate that the low-energy electronic states and
Fermi energies of carbon nanocones are strongly dependent on the nanocone geometry.

Sequentially, we want to investigate the effect of the electric field on the energy gaps of the carbon nanocones. Figure 3(a) shows the influence of $\mathbf{E}_{\|}$on the energy gaps of the five carbon nanocones. The electric field strength $E$ is increased from zero to $0.1 \gamma_{0} / \AA$. It is shown that, in the absence of the electric field, the carbon nanocones with $\alpha=60^{\circ}$ and $240^{\circ}$ are semiconducting, while these with $\alpha=120^{\circ}, 180^{\circ}$ and $300^{\circ}$ are metallic. As $E$ increases to $0.1 \gamma_{0} / \AA$, the energy gaps are strongly modulated, and ZGTs take place. It is observed that the ZGTs occur more frequently when a nanocone has a larger $\alpha$ angle and is emitted at higher strength of $E$; meanwhile, we know that both larger value of $\alpha$ and of $E$ will increase the strength of the on-site potential. Figure 3(a) confirms that the electric-field-dependent energy gap is highly sensitive to the geometric structure of a nanocone. Figure 3(b) illustrates the influence of incidence angles $\alpha_{E}$ of an electric field on the energy gap of the carbon nanocone with a disclination angle of $60^{\circ}$. At $\alpha_{E}=0^{\circ}$, two ZGTs occur at $E<0.05 \gamma_{0} / \AA$. However, as the direction of the electric field moves away from the $\hat{z}$-axis, 
the energy-gap modulation effect becomes more pronounced. It is observed that the original ZGTs at $E<0.05 \gamma_{0} / \AA$ vanish at larger values of $\alpha_{E}$. For this particular nanocone $\alpha=60^{\circ}$, the opening angle is $123.6^{\circ}$, i.e. the radius of the open edge is greater than the height of this nanocone. Therefore, the variation of the on-site potential in the radial direction is larger than that in the axial direction.
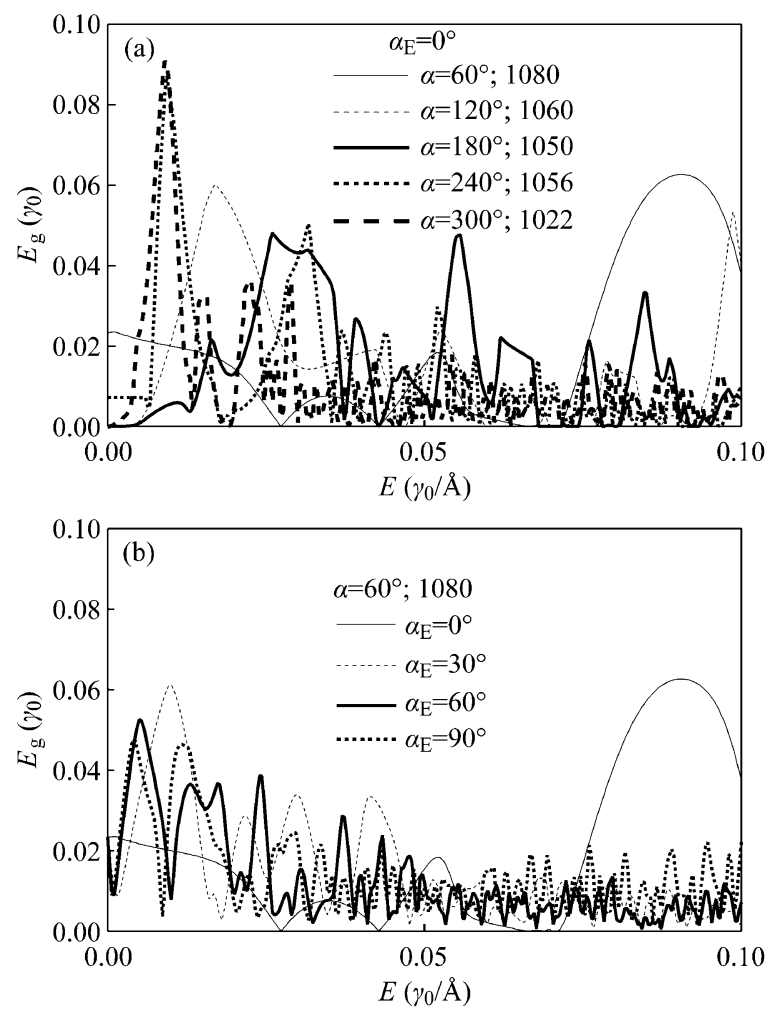

FIG. 3. (a). Influence of $\mathbf{E}=\mathbf{E}_{\|}$on electric-field-dependent energy gaps of carbon nanocones with disclination angle of $\alpha=60^{\circ}, 120^{\circ}, 180^{\circ}, 240^{\circ}$ and $300^{\circ}$, respectively. (b). Influence of electric-field incidence angle on the energy gap of the carbon nanocones with $\alpha=60^{\circ}$ and $N=1080$.

In a previous study [29], the authors showed that the energy gaps of a finite-length carbon nanotube were highly sensitive to its length. Therefore, the influence of the nanocone height, $L$, on the energy gaps is investigated in current study. Figure 4 illustrates the variation of the energy gap with $L$ for a nanocone with disclination angle $\alpha=240^{\circ}$ as a function of the strength and the direction of an applied electric field. In the absence of an electric field, the energy gap oscillates randomly with increasing $L$ and gradually approaches zero at $L>50 \AA$. The decay-like characteristic evident in Fig. 4 is very similar to that observed in finite-length carbon nanotubes with different cap configurations [29]. At $E=0.01 \gamma_{0} / \AA$, the energy-gap modulation effect is very weak in nanocones with any disclination angle when $L$ is small. However, as $L$ increases, the energy-gap modulation effect of the electric field increases significantly. Moreover, the change in the energy gap at $\alpha_{E}$ $=90^{\circ}$ is more evident than that at $\alpha_{E}=0^{\circ}$. As a result, it is shown that the energy-gap modulation effect is sensitive to changes in both the height of the carbon nanocone and the direction and strength of the electric field.

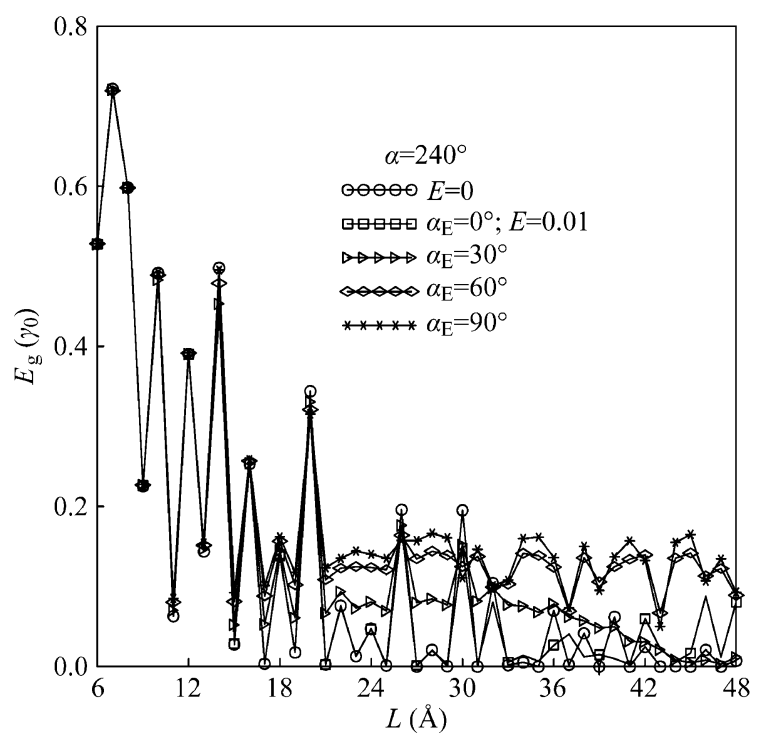

FIG. 4. Influence of nanocone height ( $L=6 \sim 48 \AA$ ) on energy gap of the carbon nanocone with $\alpha=240^{\circ}$ and $N=1056$ for $E=0 \quad \gamma_{0} / \AA$ and $E=0.01 \gamma_{0} / \AA$ which applied with the incidence angles of $0^{\circ}, 30^{\circ}, 60^{\circ}$ and $90^{\circ}$, respectively.

A single- $\pi$-band tight-binging model is used in this study to investigate the effects of an electric field on the electronic properties of perfect carbon nanocones with disclination angle of $60^{\circ}, 120^{\circ}, 180^{\circ}, 240^{\circ}$ and $300^{\circ}$, respectively. The results reveal that the low-energy electronic states, energy gap, and Fermi energy are highly sensitive to the geometric structure of the nanocone and to both the strength and direction of the applied electric field. The electric field causes a modulation of the electronic states and energy gaps of the nanocones, prompts ZGTs, and induces an almost linear change about the Fermi energy. Significantly, the HOMO and LUMO are symmetric to one another about the Fermi energy irrespective of the strength of the electric field. The present results provide further insights into the fundamental properties of conical finite-size systems in an electric field.

The authors wish to thank Professor Yun-Che Wang, (NCKU, Taiwan) for valuable assistances and suggestions. This study was supported in part by the National Science Council of Taiwan under Grant Nos. NSC 96-2221-E-492-007-MY3 and NSC 98-2221-E-006-131-MY3. We also acknowledged the National Center for Theoretical Science (NCTS) in Taiwan. 
Received 14 April 2010; accepted 19 Jun 2010; published online 6 July 2010.

\section{References}

1. S. Iijima, Nature 354, 56 (1991). doi:10.1038/354056a0.

2. J. Liu, H. Dai, J. H. Hafner, D. T. Colbert, R. E. Smalley, S. J. Tans and C. Dekker, Nature 385, 780 (1997). doi:10.1038/385780b0.

3. S. Iijima, M. Yudasaka, R. Yamada, S. Bandow, K. Suenaga, F. Kokai and K. Takahashi, Chem. Phys. Lett. 309, 165 (1999). doi:10.1016/S0009-2614(99)00642-9.

4. M. Ge and K. Sattler, Chem. Phys. Lett. 220, 192 (1994). doi:10.1016/0009-2614(94)00167-7.

5. J. C. Charlier and G. M. Rignanese, Phys. Rev. Lett. 86, 5970 (2001). doi:10.1103/PhysRevLett.86.5970.

6. S. P. Jordan and V. H. Crespi, Chem. Phys. Lett. 93, 255504 (2004).

7. M. Muñoz Navia, J. Dorantes-Dávila, M. Terrones and H. Terrones, Phys. Rev. B. 72, 235403 (2005). doi:10.1103/P hysRevB.72.235403.

8. P. E. Lammert and V. H. Crespi, Phys. Rev. B 69, 035406 (2004). doi:10.1103/PhysRevB.69.035406.

9. P. Kim, T. W. Odom, J. L. Huang and C. M. Lieber, Phys. Rev. Lett. 82, 1225 (1999). doi:10.1103/PhysRevLett. $\underline{82.1225 .}$.

10. T. W. Odom, J. L. Huang, P. Kim and C. M. Lieber, J. Phys. Chem. B 2794, 104 (2000).

11. N. G. Shang and X. Jiang, Appl. Phys. Lett. 87, 163102 (2005). doi:10.1063/1.2093919.

12. J. A. Jaszczak, G. W. Robinson, S .Dimovski and Y. Gogotsi, Carbon 41, 2085 (2003).doi:10.1016/S0008-6223 (03)00214-8.

13. G. Zhang, X. Jiang and E. Wang, Science 300, 472 (2003). doi:10.1126/science.1082264.

14. N. Muradov and A. Schwitter, Nano Lett. 2, 673 (2002). doi:10.1021/n10255909.
15. X. Zhou, H. Chen and O. Y. Zhong-can, J. Phys. Condensed Matter 13, 635 (2001). doi:10.1088/09538984/13/27/104.

16. Y. H. Kim and K. J. Chang, Phys. Rev. B 64, 153404 (2001). doi:10.1103/PhysRevB.64.153404.

17. J. O'Keeffe, C. Y. Wei and K. J. Cho, Appl. Phys. Lett. 80, 676 (2002). doi:10.1063/1.1432441.

18. Y. Li, S. V. Rotkin and U. Ravaioli, Nano Lett. 3, 183 (2003). doi:10.1021/n10259030.

19. K. H. Khoo, M. S. C. Mazzoni and S. G. Louie, Phys. Rev. B 69, 201401 (2004). doi:10.1103/PhysRevB.69.201401.

20. S. G. Kim, Y. H. Lee, P. Nordlander and D. Tománek, Chem. Phys. Lett. 264, 345 (1997). doi:10.1016/S0009-2614(96) 01328-0.

21. Y. H. Lee, S. G. Kim and D, Tománek, Chem. Phys. Lett. 265, 667 (1997). doi:10.1016/S0009-2614(96)01486-8.

22. F. L. Shyu, C. C. Tsai, M. F. Lin and C. C. Hwang, J. Phys. Soc. Jpn. 75, 104710 (2006). doi:10.1143/JPSJ.75.104710.

23. C. L. Lu, C. P. Chang, Y. C. Huang, R. B. Chen and M. L. Lin, Phys. Rev. B 73, 144427 (2006). doi:10.1103/PhysR evB.73.144427.

24. C. C. Tsai, F. L. Shyu, C. W. Chiu, C. P. Chang, R. B. Chen and M. F. Lin, Phys. Rev. B 70, 075411 (2004). doi:10.1103/PhysRevB.70.075411.

25. C. Kittle, Quantum Theory of Solid, John Wiley \& Sons, New York, 1987.

26. R. Saito, G. Dresselhaus, M. S. Dresshaus, Physical Properties of Carbon Nanotubes, Imperical College Press, London, 1998. doi:10.1142/9781860943799.

27. M. Springborg and Method of Electronic-Structure Calculations, John Wiley \& Sons, New York, 2000.

28. A. H. Castro Neto, F. Guinea, N. M. R. Peres, K. S. Novoselov and A. K. Geim, Rev. Mod. Phys. 81, 109 (2009). doi:10.1103/RevModPhys.81.109.

29. C. Chen, C. C. Tsai, J. M. Lu and C. C. Hwang, J. Phys. Chem. B 110, 12384 (2006). doi:10.1021/jp056724k. 